\title{
Modelo de lealtad a partir de un análisis de ecuaciones estructurales ${ }^{1}$
}

\section{Loyalty Model from a Structural Equation Model Analysis}

\author{
Robert Romero ${ }^{\mathrm{a}}$ \\ gilromero@usantotomas.edu.co \\ Giovanny Babativa M. ${ }^{\mathrm{b}}$ \\ josebabativa@usantotomas.edu.co
}

\begin{abstract}
Resumen
Cada día toma mayor importancia para las empresas contar con bases de datos de clientes leales, ya que el continuo seguimiento y la ampliación de negocios con ellos otorga mayor rentabilidad que la consecución de nuevos clientes. Son varias las metodologías que se han utilizado con la finalidad de medir la satisfacción y lealtad, la mayoría de ellas están basadas en la psicología conductista propia del positivismo y se apalancan en la descripción de conductas manifiestas medidas directamente y tienen como principal objetivo establecer la importancia directa que cada driver del servicio tiene sobre la lealtad; tal es el caso de las metodologías basadas en modelos de regresión. En este artículo se ejemplifica la aplicación de un modelo de lealtad que busca, a través de un análisis de ecuaciones estructurales con variables latentes, determinar el tamaño del efecto que tienen sobre la lealtad fenómenos como la satisfacción con el servicio, la intención de recompra y la recomendación.
\end{abstract}

Palabras clave: análisis factorial exploratorio, DWLS, efecto directo, efecto indirecto, modelo de medida, variable latente.

\footnotetext{
Abstract

Day by day becomes more important for companies to own a database of loyal customers, because the continuous follow up and expanding business with them gives a bigger profitability that getting new customers. Several methodologies have been used for satisfaction measure and loyalty of customers, most of them are based on Behavioural Psychology from positivist approach and leverages description of manifest behaviors measured directly and whose main objective is establish the direct

${ }^{1}$ Romero, R., Babativa, G. Modelo de lealtad a partir de un análisis de ecuaciones estructurales. Comunicaciones en Estadística, 9(2), 165-197.

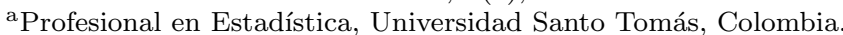

${ }^{\mathrm{b}}$ Decano Facultad de Estadística, Universidad Santo Tomás, Colombia.
} 
importance that each service driver has about loyalty, as in case of methodologies based on regression models. In this paper I illustrate an application of a Loyalty Model that looking for through an analysis of Structural Equation Modeling with latent variables, define the size of effect on the loyalty for phenomena like overall satisfaction with service, repurchase intention and recommendation.

Keywords: direct effect, DWLS, exploratory factor analysis, indirect effect, latent variable, measure model.

\section{Introducción}

Para una compañía siempre ha sido importante la relación con sus clientes; por ello en diferentes trabajos se han planteado patrones para describir dicha asociación. En esta búsqueda ha cobrado relevancia el uso de modelos estadísticos que permiten establecer la forma en que interactúan las distintas variables que determinan el comportamiento de un cliente.

Entre los modelos utilizados para describir la relación entre las variables componentes de la satisfacción y la lealtad se encuentran los modelos de regresión, análisis factoriales y últimamente los modelos de ecuaciones estructurales (SEM); los cuales, tienden a ser los más adecuados para realizar la estimación debido a que algunas de estas variables son exógenas y endógenas al mismo tiempo y no es fácil establecer una relación directa entre ellas. En este caso el modelo de ecuaciones estructurales permite establecer los efectos directos e indirectos entre las variables; dichos valores son utilizados para construir un indicador que permita establecer estrategias para elevar el grado de lealtad de sus clientes.

En este artículo se explora la utilización de los modelos de ecuaciones estructurales (SEM) con el propósito de construir un modelo de lealtad a partir de los componentes del servicio ofrecido al cliente. En una primera parte se describe la teoría general de los modelos SEM; en la segunda parte se realiza el análisis descriptivo de los datos y se examinan las posibles relaciones entre las variables utilizadas; por último se presenta un ejemplo práctico de la estimación con modelos SEM y sus respectivos resultados y se establecen las conclusiones y futuras líneas de investigación.

\section{Conceptos fundamentales}

En la actualidad el análisis de modelos SEM es una importante técnica multivariada que se aplica en diferentes campos profesionales; no obstante, las técnicas estadísticas que la soportan no son de reciente aparición, y tuvo que transcurrir bastante tiempo antes de que se pudiera explotar su potencial, esto gracias a los avances computacionales, que como en muchos otros campos han sido vía arteria del desarrollo de conocimiento; de igual manera, permitieron una investigación 
más profunda de la técnica, la cual se ha apalancado principalmente en estudios de simulación.

A grandes rasgos, los modelos SEM permiten la representación de una serie de hipótesis de relaciones (principalmente lineales) entre una serie de variables medidas y causadas a su vez por una diversidad de fenómenos subyacentes, los cuales no son directamente observables, que para este caso se denominan latentes (en otros campos de investigación se conocen como factores o constructos). Las variables latentes son de gran importancia en muchas disciplinas pero carecen de un modo preciso de medición, en lo relativo a su existencia o influencia en otros fenómenos o variables.

Posibles ejemplos de variables latentes pueden ser la calidad del aire, la felicidad o la inteligencia, fenómenos que, dada su inobservabilidad, no se pueden medir directamente, razón por la cual, los investigadores definen una serie de herramientas operacionales a través de las cuales se puedan construir de manera indirecta. A estas herramientas se le llama variables manifiestas (observadas) y en la metodología los modelos SEM sirven como indicadoras del fenómeno subyacente que representan.

El término SEM es una generalización para varios tipos de modelos y estadísticamente representan una extensión de procedimientos de modelos lineales generales (MLG), tales como ANOVA, análisis de regresión múltiple y análisis factorial, que tienen las siguientes características diferenciadoras de las técnicas de modelización clásica:

- Generalmente se conciben como construcciones teóricas de fenómenos que no son medibles directamente.

- Toman en cuenta los posibles errores de medición de las variables con las cuales se construyen los factores latentes. Las varianzas de los términos de error son los parámetros a estimar al momento de ajustar el modelo, por lo que en este caso es correcto llamarlo análisis de estructura de covarianzas.

- Los modelos son ajustados a partir de matrices de índices de interrelación (matriz de correlaciones o covarianzas), aunque algunas veces también se realizará el análisis sobre las medias de las variables.

Entre los tipos de modelos SEM se puede mencionar:

- Path Analysis: este tipo de análisis busca, mediante el apoyo de un path diagram, descomponer la covarianza entre las variables del modelo con el fin de establecer la medida de relación entre los efectos causales y las medidas de covariación. Dichas relaciones pueden ser directas, espurias, indirectas o conjuntas. Adicionalmente permite medir el efecto causal (directo, indirecto y total) que una variable tiene sobre otra en el modelo. Según Long (1983) también son llamados modelos de estructura de covarianzas y se descomponen en dos: el modelo de componente estructural y el modelo de componente de medida. 
- Análisis factorial confirmatorio: permite analizar los patrones de relación o causación entre variables latentes (constructos) del modelo estructural, con el fin de verificar si este es válido o si sus interrelaciones generan alguna interpretación plausible.

- Modelos de curva de crecimiento: los anteriores modelos citados se basan en datos de corte transversal obtenidos a través de una muestra de individuos en un punto de tiempo $t$. Los modelos de curva de crecimiento permiten analizar para datos de corte longitudinal, la dinámica de los cambios y evoluciones del comportamiento de los procesos bajo estudio.

\section{Path Diagram (diagrama de ruta)}

A veces los sistemas de ecuaciones son muy complejos y requieren introducir muchas relaciones entre las variables; en este caso generalmente se prefiere la representación gráfica del modelo en consideración mediante un diagrama causal o Path Diagram, como el de la figura 1; este tipo de representación equivale a un conjunto de ecuaciones que configuran el modelo. En la representación gráfica se usa una notación especial.

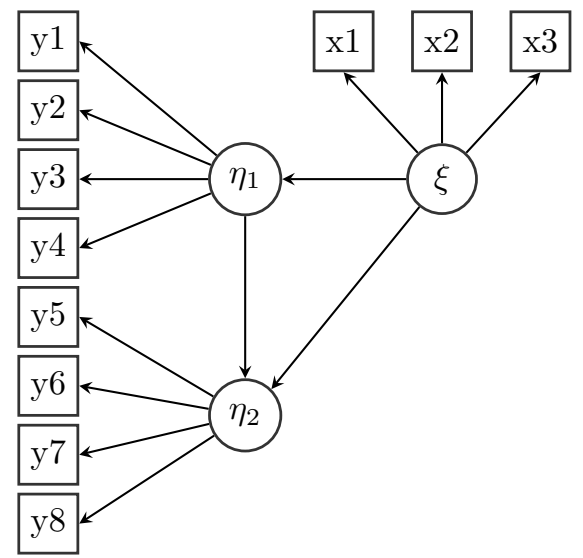

Figura 1: Modelo de ecuación estructural representado por un Path Diagram. Fuente: elaboración propia.

Cuando el modelo incluye tanto variables observadas como latentes, las primeras se representan con cuadrados o rectángulos y las segundas con círculos o elipses; las flechas que salen de las variables latentes a las observadas se llaman relaciones de medición. Las variables observadas están afectadas por un término de error aleatorio, el cual es representado en el diagrama por una flecha unidireccional que apunta a la variable observable. La covariación entre dos errores de medición es representada por una flecha bidireccional que los une. La relación entre variables 
se indica por una flecha unidireccional desde la variable exógena a la variable endógena (tabla 1).

Tabla 1: Símbolos usados comúnmente en análisis SEM. Fuente: elaboración propia.

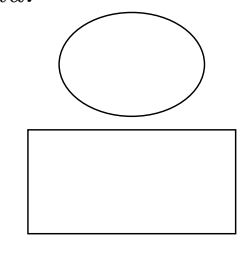

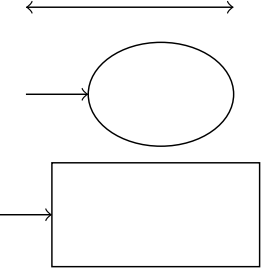

$\mathrm{O}$

$\mathrm{O}$

$\mathrm{O}$

0

$\mathrm{O}$

$\mathrm{O}$
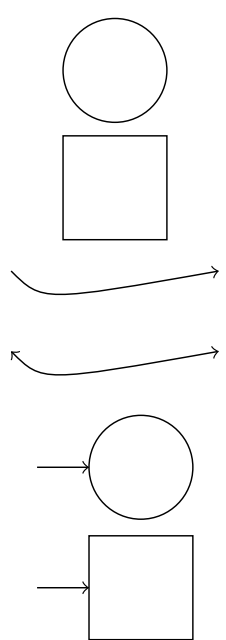

Variable latente

Variable

observada

Relación entre

dos variables

Covarianza entre dos variables

Medida de error de la variable latente

Medida de Error de la variable observada

\section{Path Analysis}

Ya en la sección 2 se determinó el principal objetivo del Path Analysis; para desarrollar por completo el concepto se detallarán los tipos de relación que puede llevar a que dos variables $x$ y $y$ covaríen.

1. $x$ y $y$ pueden covariar si $y$ tiene algún efecto sobre $x$ (o al contrario), como la relación representada por un modelo de regresión simple, presente en la figura 2:

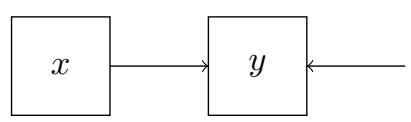

Figura 2: Path Diagram regresión simple. Fuente: elaboración propia.

Estas relaciones se llaman directas, aunque también pueden ser recíprocas (figura 3): 


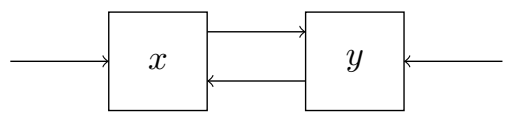

Figura 3: Path Diagram relación recíproca. Fuente: elaboración propia.

2. $x$ y y covarían si tienen una causa común $z$; este tipo de relación se denomina espúrea (figura 4):

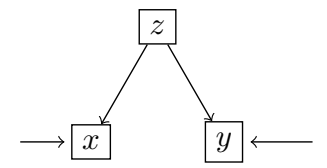

Figura 4: Path Diagram relación espuria. Fuente: elaboración propia.

3. $x$ y y también covarían si están relacionadas a través de una tercera variable $z$; este tipo de relación se denomina indirecta (figura 5):

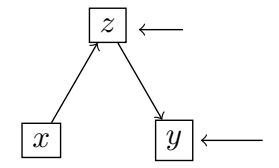

Figura 5: Path Diagram relación indirecta. Fuente: elaboración propia.

Una vez señalados los posibles tipos de covariación entre las variables del modelo, se procede ahora a implantar una serie de reglas de descomposición que permitan establecer las relaciones entre las covariaciones y los parámetros de este; una vez fijadas estas relaciones, se podrá realizar a partir de ellas el cálculo de estimación de parámetros. Es importante no perder de vista que el ajuste del modelo se realiza sobre las matrices de covarianzas de las variables observadas, las cuales son previamente centradas para dejar de un lado el efecto que pueda tener la media de cada variable observada.

Las varianzas y covarianzas de las variables observadas son en sí mismas medidas iniciales del modelo. Según Batista \& Coenders (2000), para derivar los demás parámetros se sigue que:

1. La covarianza entre dos variables se calcula como la suma entre los efectos directos, indirectos, espurios y conjuntos. Cada uno de estos representa en el Path Diagram una posible manera de unir las variables. El efecto se calcula como el producto de la varianza de la variable de partida por todos los parámetros asociados a las 
flechas recorridas hasta llegar a unir las dos variables de interés (figura 6 y figura 7 ).

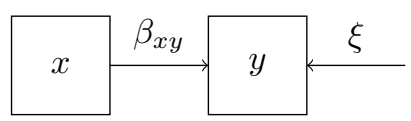

Figura 6: Efecto total de $x$ sobre y. Fuente: elaboración propia.

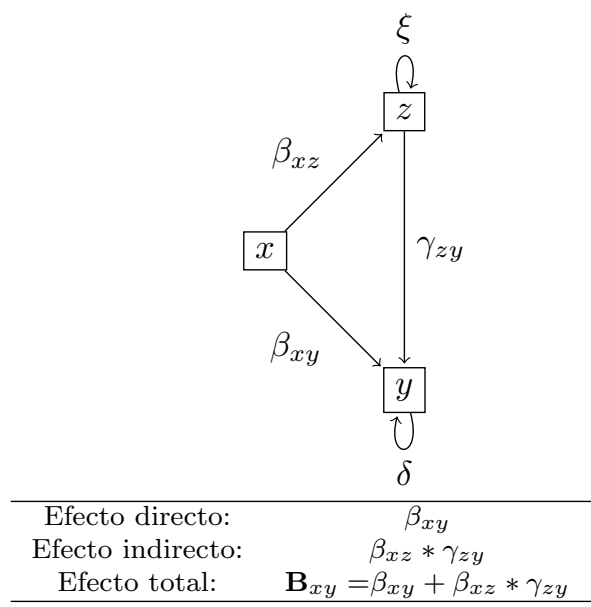

Figura 7: Descomposición del efecto de $x$ sobre y. Fuente: elaboración propia.

2. La varianza de una variable exógena se calcula como la varianza del término de error más la varianza explicada por otras variables en el modelo. Asimismo, la varianza explicada puede expresarse como una función de todas las exógenas con efecto directo sobre la variable endógena, como la subida de la totalidad de productos entre los efectos directos y las covarianzas entre las variables endógena y exógenas relacionadas por los efectos.

Aplicando las anteriores reglas, se obtiene un sistema de ecuaciones estructurales, el cual expresa la matriz de covarianzas en función de los parámetros del modelo,

$$
\Sigma=\Sigma(\theta)
$$

Donde $\theta$ es un vector que contiene los parámetros del modelo (efectos directos, indirectos, varianzas, covarianzas de errores, de perturbaciones y de variables exógenas). 


\section{Etapas de construcción de los modelos SEM}

Como se ha visto, el análisis de modelos SEM requiere de un conocimiento previo de las posibles interacciones o relaciones entre las variables del modelo tanto exógenas como endógenas; esto quiere decir que en el Path Diagram se grafican las hipotéticas relaciones de los fenómenos bajo estudio. A partir de este punto se enumera una serie de pasos ${ }^{1}$ que debe seguir el investigador antes de aplicar el modelo en la práctica; estos pasos son iterativos, ya que dependiendo de lo que pase en alguno de ellos, se debe retornar al primero:

1. Especificación

2. Identificación

3. Recogida de datos

4. Estimación

5. Diagnóstico

6. Evaluar si el modelo es adecuado (no cumple: vuelva a paso 1)

7. Uso

\section{Aplicación para el modelo de lealtad}

Tal vez una de las inquietudes más frecuentes de las empresas, sin importar el país de origen, es conocer qué tan leales son sus clientes; para este fin se han construido múltiples tipos de modelos que abordan temáticas que van desde el capital de marca hasta la evaluación de las dimensiones del servicio final ofrecido.

El presente artículo toma como línea de base la simulación de una investigación sobre la calidad en la atención recibida por el usuario en una empresa prestadora de servicios. Es importante tener en cuenta que a diferencia de la evaluación de productos físicos, la evaluación del servicio se basa en componentes intangibles, los cuales están sujetos a la subjetividad del usuario según la experiencia vivida (Saurina 1997).

Para ello se tomó una medida de satisfacción con el servicio recibido, mediante las siguientes variables:

- intenc: la intención de tomar nuevamente los servicios de la empresa.

- recom: la disposición a recomendar la empresa a otras personas.

- horario: los horarios de atención.

- ubicacion: la ubicación de las sedes en el lugar donde se necesita.

${ }^{1}$ Para una ilustración detallada de estos pasos ver Romero (2015). 
- comodidad: la comodidad de las sedes.

- senaliza: la señalización en el interior de las sedes.

- seguridad: la seguridad que siente en las sedes.

- personal: cantidad de personas atendiendo.

- tiempo: tiempo de espera en área de recaudo.

- amabi: amabilidad y respeto recibido en recaudo.

- agili: agilidad de la persona que atiende en recaudo.

- manejo: disposición de las filas en recaudo.

- espera: tiempo de espera al asesor.

- respet: amabilidad y respeto del asesor.

- interes: interés demostrado por el asesor.

- claro: claridad de la información que brindó el asesor.

- soluc: solución efectiva a las necesidades.

- $a g$ : agilidad en la respuesta recibida.

- cupos: los cupos otorgados.

- requisit: los requisitos exigidos para adquirir el servicio.

- trami: la agilidad en los trámites.

- antigue: el reconocimiento de la antigüedad como cliente.

- comporta: el reconocimiento por el buen uso del servicio.

- acompa: el servicio posventa.

- nuevo: el ofrecimiento de nuevos productos.

Dichas variables se miden en una escala ordinal de uno a diez, siendo 1 la menor calificación y 10 la mayor. El enfoque del artículo será realizar el cálculo de un indicador de lealtad del cliente a partir del método de ecuaciones estructurales, el cual permitirá descomponer los efectos tanto directos como indirectos que sobre la lealtad tienen las diferentes dimensiones latentes del servicio. Previo a realizar el estudio estructural, se hace un análisis factorial exploratorio con el fin de encontrar las relaciones subyacentes entre las variables observadas.

El modelo teórico que se va a exponer contempla que las variables anteriormente citadas conforman dimensiones de servicio las cuales tienen un efecto indirecto en la lealtad y directo en la satisfacción, lo cual a su vez afecta directamente la lealtad del cliente. 


\subsection{Análisis exploratorio de las variables}

Después de haber enumerado las variables con las cuales se va a calcular el indicador de lealtad mediante el modelo global de ecuaciones estructurales y teniendo en cuenta su carácter ordinal, en esta sección se muestran los resultados de la aplicación de las pruebas de normalidad. La escala para cada una de las variables se encuentra entre 1 y 10, la cual es suficientemente amplia para que ser tratadas como variables numéricas (aunque no continuas), razón por la cual es válido realizar análisis y pruebas estadísticas con el fin de verificar los supuestos de los modelos.

Para realizar las pruebas de normalidad se usa el valor $p$ asociado al estadístico de Anderson-Darling (tabla 2), teniendo en cuenta que su hipótesis nula es:

$$
H_{0}: X \sim N(\mu, \sigma)
$$

Tabla 2: Test de Anderson Darling para contraste de normalidad. Fuente: elaboración propia.

\begin{tabular}{|c|c|c|}
\hline Variable & Estadística & Valor $p$ \\
\hline horario & 631,5722 & 0,00 \\
\hline ubicacion & 733,9707 & 0,00 \\
\hline comodidad & 545,6445 & 0,00 \\
\hline senaliza & 609,0171 & 0,00 \\
\hline seguridad & 668,7004 & 0,00 \\
\hline personal & 250,9835 & 0,00 \\
\hline tiempo & 236,6273 & 0,00 \\
\hline amabi & 802,5184 & 0,00 \\
\hline agili & 391,6763 & 0,00 \\
\hline manejo & 391,1865 & 0,00 \\
\hline espera & 338,9154 & 0,00 \\
\hline respet & 876,6862 & 0,00 \\
\hline interes & 630,6738 & 0,00 \\
\hline claro & 684,7826 & 0,00 \\
\hline soluc & 603,0671 & 0,00 \\
\hline ag & 585,1464 & 0,00 \\
\hline cupos & 504,0901 & 0,00 \\
\hline requisit & 453,0338 & 0,00 \\
\hline trami & 459,0459 & 0,00 \\
\hline antigue & 474,4710 & 0,00 \\
\hline comporta & 509,7517 & 0,00 \\
\hline acompa & 432,8783 & 0,00 \\
\hline nuevo & 404,6410 & 0,00 \\
\hline
\end{tabular}

Comunicaciones en Estadística, agosto 2016, Vol. 9, No. 2 
Tabla 3: Test de normalidad multivariada de Mardia. Fuente: elaboración propia.

\begin{tabular}{|c|c|}
\hline g1p: & 103,7106 \\
chi.skew: & 142809,5 \\
p.value.skew: & 0,00 \\
\hline g2p: & 1320,195 \\
z.kurtosis: & 998,6971 \\
p.value.kurt: & 0,00 \\
\hline chi.small.skew: & 142865,7 \\
p.value.small: & 0,00 \\
\hline
\end{tabular}

En este caso el test rechaza la hipótesis de normalidad para cada variable observada; igualmente los resultados de la prueba de Mardia presentados en la tabla 3 rechazan la hipótesis de normalidad multivariada; sin embargo, los modelos de ecuaciones estructurales manejan métodos robustos para variables no normales, por lo cual es viable su aplicación.

A fin de analizar la consistencia de los datos, se utilizó el coeficiente Alpha de Cronbach, el cual estima la fiabilidad de la información a través del conjunto de ítems que se midieron. El procedimiento general para el cálculo del Alpha de Cronbach parte de la matriz de correlaciones de Pearson, ya que la utilización de una escala Likert con una cantidad mayor a 6 categorías estabiliza el coeficiente (Gelin et al. 2003) (tabla 4).

Tabla 4: Alpha de Cronbach. Fuente: elaboración propia.

\begin{tabular}{|c|c|c|}
\hline alpha & std.alpha & Guttman's Lambda 6 \\
\hline 0,96 & 0,96 & 0,97 \\
\hline
\end{tabular}

El coeficiente Alpha de Cronbach es de 0.96 , por lo que se concluye que las variables recogen con alta fiabilidad la información requerida (Streiner 2003).

\subsection{Análisis factorial exploratorio}

Antes de entrar en la etapa de construcción del modelo, es necesario dar coherencia a la diversidad de información que se está midiendo mediante alguna técnica que permita encontrar, a partir de su estructura de correlación, relaciones subyacentes entre los vectores de análisis, con el fin de definir grupos de variables que estén altamente correlacionadas entre sí a $k$ factores latentes que expliquen la mayor cantidad de varianza de la matriz $\mathbf{X}$ original. Existen diversas técnicas estadísticas de interdependencia, en este caso se utiliza el análisis factorial exploratorio (EFA).

A pesar de que el EFA se basa en el supuesto de normalidad, se considera su uso en este artículo ya que el objetivo previo a la especificación del modelo estructural es contextualizar la situación y tener un modelo de medición que proporcione una base para un análisis causal de relaciones entre variables latentes (Loehlin 2004). 


\subsection{Tratamiento de variables ordinales}

El paso previo para la realización de un EFA es hacer una evaluación de la matriz de correlaciones con el fin de establecer si se justifica su aplicación; sin embargo, se debe tener en cuenta que en este caso se está tratando con variables discretas ordinales, por lo que se debe estudiar la conveniencia de usar la matriz de correlaciones de Pearson, ya que en algunas ocasiones no es apropiada para el análisis; en estos casos son las llamadas correlaciones policóricas las que deben emplearse como punto de partida (Olsson 1979). Este tipo de correlaciones se usa para relacionar características que, aunque en principio son continuas, pero en las que se utilizó una escala ordinal para medirlas; un claro ejemplo son las características medidas mediante las escalas de Likert utilizadas en este artículo. Coenders et al. (1979) afirman que:

Un enfoque típico para modelar variables ordinales es asumir que para cada variable ordinal $y_{i}$ hay una variable subyacente $y_{i}^{*}$, y que cada $y_{i}$ se relaciona a $y_{i}^{*}$ a través de la función de paso:

$$
y_{i}=k \text { cuando } \tau_{i k-1}<y_{i}^{*} \leq \tau_{i k}
$$

Para $k=1, \ldots, m_{i}$, donde $\tau_{i}=-\infty, \tau_{i k}<\tau_{i k+1}, \tau_{i m i}=\infty$. Los parámetros $\tau_{i}$ con $i=1, \ldots, m_{i-1}$, son llamados umbrales de la $i$-ésima variable.

Sin embargo, dependiendo del método de estimación, la matriz de correlaciones policóricas puede ser no definida positiva, con lo que el análisis factorial no sería posible; así, tomando como apoyo computacional el paquete psych del entorno $\mathrm{R}$, se analiza por pruebas de bootstrap a las matrices de correlación de Pearson y policóricas para determinar con cuál de ellas se realiza el EFA. En esta simulación los casos se crean mediante MASR y las correlaciones se calculan tantas veces como iteraciones hay. La media de correlación y su respectiva desviación estándar son calculadas con base en la transformación $Z$ de Fisher de las correlaciones.

Si se denota por $\hat{\rho}$ a la correlación policórica de interés, el intervalo de la transformación de Fisher estará definido por:

$$
z(\hat{\rho}) \pm z_{\delta / 2} * S E(\hat{\rho}) /\left(1-\hat{\rho}^{2}\right)
$$

Donde

$$
z(\hat{\rho})=0.5 * \ln [(1+\rho) /(1-\rho)]
$$

y en el que $S E(\hat{\rho})$ es el error estándar de la correlación policórica (Hoyle 2012).

Los resultados se muestran en la figura 8:

Comunicaciones en Estadística, agosto 2016, Vol. 9, No. 2 


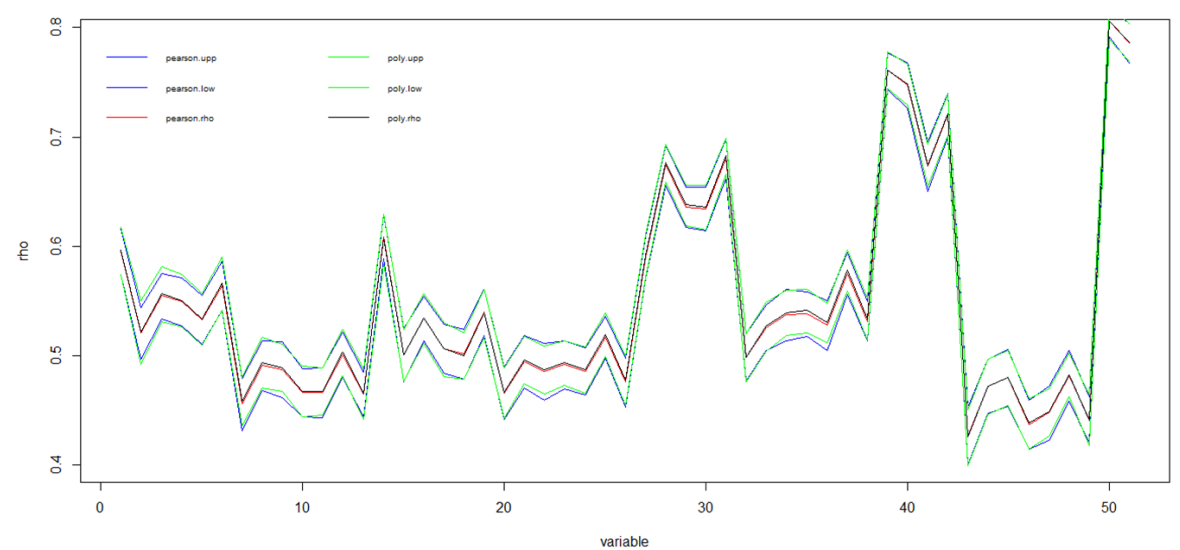

Figura 8: CI (95\%) generados por Bootstrap. Fuente: elaboración propia

En la figura 8 se observa que los resultados generados por el método de correlaciones policóricas y la matriz de correlación de Pearson para datos continuos son muy similares entre sí; a esto se suma la posibilidad de obtener una matriz de correlación policórica no definida positiva define que el presente artículo se decante por la utilización de la correlación clásica de Pearson para la realización del análisis factorial exploratorio.

\subsection{Número de factores a retener}

Como regla inicial, el número de factores a retener debe ser cercano al número de valores propios positivos de la matriz de correlación (Field 2000); sin embargo en este caso es posible obtener una gran cantidad de valores propios positivos pero muy cercanos a cero, lo que en algunas ocasiones hace que se maneje gran cantidad de factores que aportan muy poca información de análisis; en este caso se utiliza la regla de Very Structure Simple, según los pasos citados por Romero (2015).

Los resultados obtenidos por el método VSS con asistencia del paquete psych del entorno $R$ muestran que el número de óptimo a retener es de cuatro factores, ya que en este punto se encuentra el mayor número de factores con los que $V S S_{v k} \approx 1$ (figura 9). 
Very Simple Structure

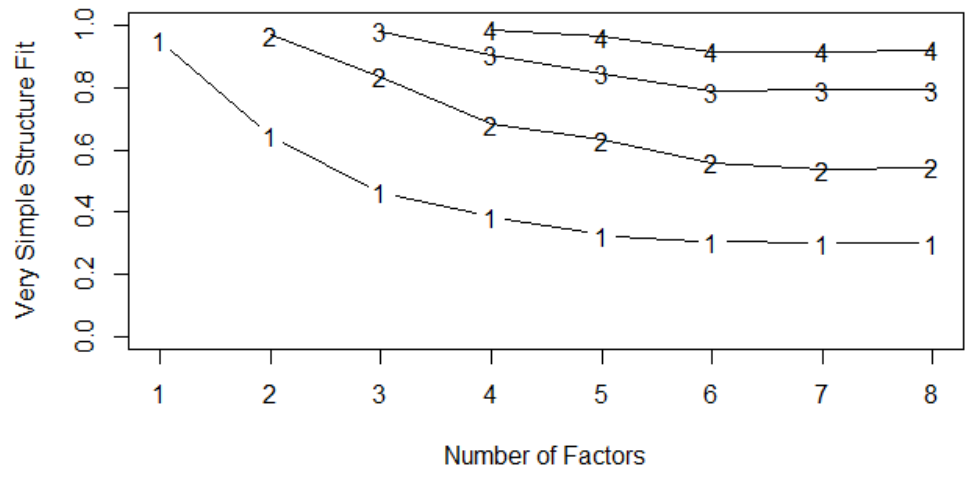

Figura 9: Factores a retener por la regla VSS. Fuente: elaboración propia.

El método usado para la extracción de los factores es el de factor principal, en el cual se extrae la matriz factorial con la propiedad de que los factores explican la máxima varianza y adicionalmente son incorrelacionados; en este se supone que existe un factor común subyacente a las variables por lo que busca extraer la máxima varianza en cada factor para que al final los $k$ factores resultantes expliquen la mayor cantidad de varianza común, aunque las cargas de los factores extraídos no difieren sustancialmente del método de la componente principal (Rietveld \& Van Hout 1993).

A este método se le aplicó la rotación Varimax, con el fin de maximizar los pesos de cada factor, esperando así que cada variable sea bien representada en solo uno de ellos y a la vez se minimice el máximo número de variables correlacionadas a cada factor.

El análisis resultante extrayendo los cuatro factores mediante el método citado se expone en las tablas 5, 6 y 7 :

Comunicaciones en Estadística, agosto 2016, Vol. 9, No. 2 
Tabla 5: Resultados del análisis factorial exploratorio. Fuente: elaboración propia.

\begin{tabular}{|c|c|c|c|c|c|c|}
\hline Ítem & FAC1 & FAC2 & FAC3 & FAC4 & Comunalidad & Unicicidad \\
\hline antigue & & 0,78 & & & 0,75 & 0,25 \\
comporta & & 0,78 & & & 0,75 & 0,25 \\
nuevo & & 0,73 & & & 0,68 & 0,32 \\
cupos & & 0,73 & & & 0,66 & 0,34 \\
acompa & & 0,72 & & & 0,72 & 0,28 \\
requisit & & 0,67 & & & 0,65 & 0,35 \\
trami & & 0,67 & & & 0,65 & 0,35 \\
claro & & & 0,79 & & 0,81 & 0,19 \\
interes & & & 0,76 & & 0,82 & 0,18 \\
ag & & 0,76 & & 0,8 & 0,2 \\
soluc & & & 0,74 & & 0,76 & 0,24 \\
respet & & & 0,7 & & 0,69 & 0,31 \\
espera & 0,46 & & 0,49 & & 0,64 & 0,36 \\
tiempo & 0,79 & & & & 0,81 & 0,19 \\
personal & 0,71 & & & & 0,73 & 0,27 \\
agili & 0,66 & & & & 0,71 & 0,29 \\
manejo & 0,65 & & & & 0,7 & 0,3 \\
senaliza & & & & 0,65 & 0,66 & 0,34 \\
seguridad & & & & 0,6 & 0,56 & 0,44 \\
horario & & & & 0,57 & 0,51 & 0,49 \\
comodidad & & & & 0,45 & 0,43 & 0,37 \\
ubicacion & & & & & 0,58 & 0,46 \\
amabi & & & & & & \\
\hline
\end{tabular}

Tabla 6: Estadísticos de resumen. Fuente: elaboración propia.

\begin{tabular}{|l|c|c|c|c|}
\hline Item & FAC1 & FAC2 & FAC3 & FAC4 \\
\hline SS loadings & 4,99 & 4,26 & 3,32 & 3,14 \\
Proportion Var & 0,22 & 0,19 & 0,14 & 0,14 \\
Proportion Explained & 0,32 & 0,27 & 0,21 & 0,2 \\
Correlation of scores with factors & 0,94 & 0,94 & 0,9 & 0,84 \\
Multiple R square of scores with factors & 0,88 & 0,88 & 0,82 & 0,7 \\
\hline
\end{tabular}

En el análisis de la etapa exploratoria se encuentra un total de cuatro factores que recogen un $68 \%$ de la varianza; dichos factores generan las dimensiones subyacentes del servicio según las evaluaciones realizadas a los usuarios. Estas dimensiones diferencian claramente cada uno de los momentos de atención que se presentan en las empresas de servicios.

1. Primer factor: el primer factor es conformado por un total de siete variables con ponderaciones mayores a 0,65 ; a su vez estas variables no alcanzan en otros factores importancias mayores a 0,25 , todas estas variables se refieren a la satisfacción con el trato de servicios ofrecido por la empresa. 
Tabla 7: Dimensiones y las variables asociadas. Fuente: elaboración propia.

\begin{tabular}{|c|c|}
\hline Dimensión & Variable \\
\hline Trato & $\begin{array}{l}\text { antigue: el reconocimiento de la antigüedad como cliente. } \\
\text { comporta: el reconocimiento por el buen uso del servicio. } \\
\text { nuevo: el ofrecimiento de nuevos productos. } \\
\text { cupos: los cupos otorgados. } \\
\text { acompa: el servicio posventa. } \\
\text { requisit: los requisitos exigidos para adquirir el servicio. } \\
\text { trami: la agilidad en los trámites. }\end{array}$ \\
\hline Asesores & $\begin{array}{l}\text { claro: la claridad de la información que le brindó el asesor. } \\
\text { interes: el interés que demostró por sus necesidades. } \\
\text { ag: la agilidad en la respuesta. } \\
\text { soluc: la Solución efectiva a sus requerimientos. } \\
\text { respet: la amabilidad y respeto en el trato del asesor. } \\
\text { espera: el tiempo de espera previo a la atención del asesor. }\end{array}$ \\
\hline Cajas & $\begin{array}{l}\text { tiempo: el tiempo de espera en las filas. } \\
\text { personal: la cantidad de personas que atienden las cajas. } \\
\text { agili: la agilidad de las personas que atienden las cajas. } \\
\text { manejo: el manejo adecuado de las filas para clientes. }\end{array}$ \\
\hline Oficinas & $\begin{array}{l}\text { senaliza: la señalización en el interior de las oficinas. } \\
\text { seguridad: la seguridad que siente en la oficina. } \\
\text { horario: los horarios de atención de las oficinas. } \\
\text { comodidad: la comodidad de las oficinas. } \\
\text { ubicacion: la ubicación de las oficinas en el lugar donde se necesita. } \\
\text { amabi: la amabilidad y respeto de las personas que atienden. }\end{array}$ \\
\hline
\end{tabular}

Comunicaciones en Estadística, agosto 2016, Vol. 9, No. 2 
2. Segundo factor: está conformado por seis variables asociadas a la atención personal recibida por los asesores de servicio cuyas ponderaciones son mayores a 0,49 .

3. Tercer factor: compuesto por cuatro variables con ponderaciones mayores a 0,65 y está conformado por las variables que evalúan la atención recibida en cajas.

4. Cuarto factor: lo integran seis variables que tienen ponderaciones mayores a 0,45 y conforman la dimensión subyacente de atención en oficinas.

\subsection{Etapa de especificación}

En esta sección se busca establecer formalmente el modelo; anteriormente se establecieron cuatro configuraciones de interdependencia, en las que los factores son medidas latentes de la satisfacción con cada uno de los momentos del servicio y en las que se asume la existencia de una relación lineal entre los factores y las variables manifiestas. Es así que el primer factor (Oficinas) toma la forma presentada en la figura 6.5.

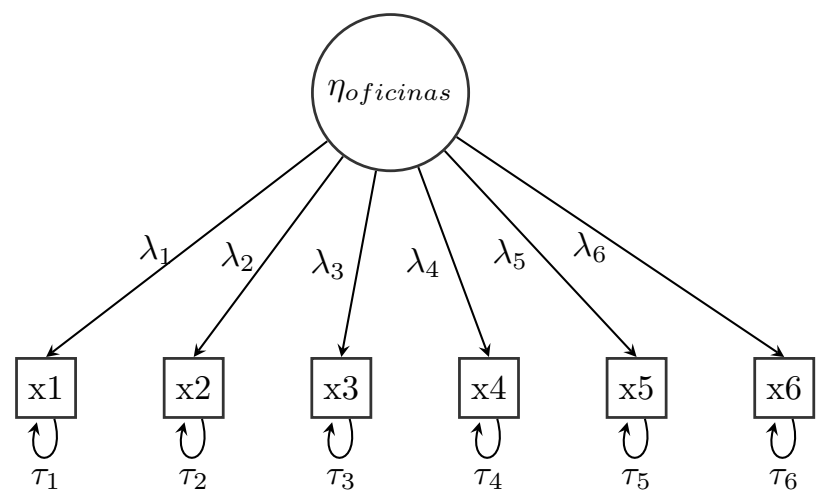

Figura 10: Path Diagram factor 1 - Oficinas. Fuente: elaboración propia.

El Path Diagram expresa el efecto directo que tiene la variable subyacente de satisfacción con las oficinas sobre las variables manifiestas, que se pueden expresar en el modelo de medida:

$$
\begin{gathered}
\mathbf{X}=\Lambda \eta+\tau \\
{\left[\begin{array}{l}
x_{1} \\
x_{2} \\
x_{3} \\
x_{4} \\
x_{5} \\
x_{6}
\end{array}\right]=\left[\begin{array}{c}
\lambda_{1} \\
\lambda_{2} \\
\lambda_{3} \\
\lambda_{4} \\
\lambda_{5} \\
\lambda_{6}
\end{array}\right][\eta]+\left[\begin{array}{c}
\tau_{1} \\
\tau_{2} \\
\tau_{3} \\
\tau_{4} \\
\tau_{5} \\
\tau_{6}
\end{array}\right]}
\end{gathered}
$$


Donde $\eta$ es la variable latente Oficinas, $\Lambda$ es la matriz de efectos desconocidos a estimar, $\tau$ es la matriz de error de medida y finalmente $\mathbf{X}$ es la matriz de variables observadas, donde $x_{1}=$ senaliza, $x_{2}=$ seguridad, $x_{3}=$ horario, $x_{4}=$ comodidad, $x_{5}=$ ubicacion y $x_{6}=$ amabi. La especificación de los demás factores se realiza de manera análoga.

Para finalizar la etapa de especificación no se debe perder de vista que los anteriores factores latentes determinados de manera exploratoria tienen efecto (directo e indirecto) sobre la satisfacción del servicio, por lo que en esta etapa se definen las siguientes hipótesis estructurales:

- H1: el factor subyacente oficinas tiene un efecto significativo sobre la satisfacción con el servicio, es decir, $\eta_{\text {oficinas }} \neq 0$.

- H2: el factor subyacente cajas tiene un efecto significativo sobre la satisfacción con el servicio, es decir, $\eta_{\text {cajas }} \neq 0$.

- H3: el factor subyacente asesores tiene un efecto significativo sobre la satisfacción con el servicio, es decir, $\eta_{\text {asesores }} \neq 0$.

- H4: el factor subyacente trato tiene un efecto significativo sobre la satisfacción con el servicio, es decir, $\eta_{\text {trato }} \neq 0$.

- H5: la satisfacción general tiene un efecto significativo sobre la lealtad con la marca, es decir, $\beta_{\text {sat }_{\text {gen }}} \neq 0$.

- H6: la recomendación tiene un efecto significativo sobre la lealtad con la marca, es decir, $\beta_{\text {recom }} \neq 0$.

- H7: la intención de recompra tiene un efecto significativo sobre la lealtad con la marca, es decir, $\beta_{\text {intenc }} \neq 0$.

En este caso la satisfacción general está dada por el diagrama presente en la figura 11:

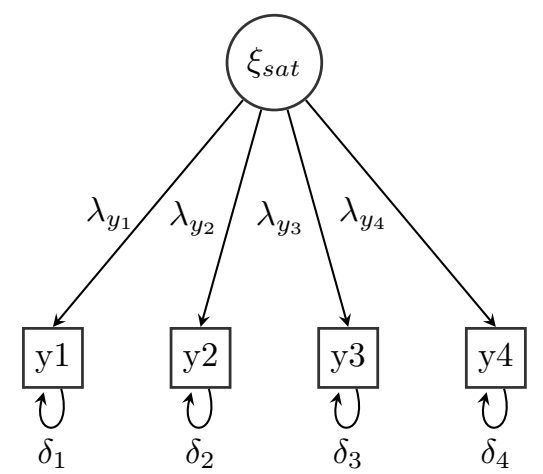

Figura 11: Path Diagram factor Satisfacción. Fuente: elaboración propia. 
Y la ecuación que representa su modelo de medida se expresa así

$$
\left[\begin{array}{l}
y_{1} \\
y_{2} \\
y_{3} \\
y_{4}
\end{array}\right]=\left[\begin{array}{l}
\lambda_{y_{1}} \\
\lambda_{y_{2}} \\
\lambda_{y_{3}} \\
\lambda_{y_{4}}
\end{array}\right][\xi]+\left[\begin{array}{l}
\delta_{1} \\
\delta_{2} \\
\delta_{3} \\
\delta_{4}
\end{array}\right]
$$

Donde $\xi$ es la variable latente de satisfacción general, $\Lambda$ es la matriz de efectos desconocidos a estimar, $\delta$ es la matriz de error de medida y finalmente $\mathbf{Y}$ es la matriz de variables observadas, donde $y_{1}=$ planta física, $y_{2}=$ área financiera, $y_{3}=$ atención personal, $y_{4}=$ variedad de productos. En la figura 12 se observa el path de la especificación completa del modelo: 


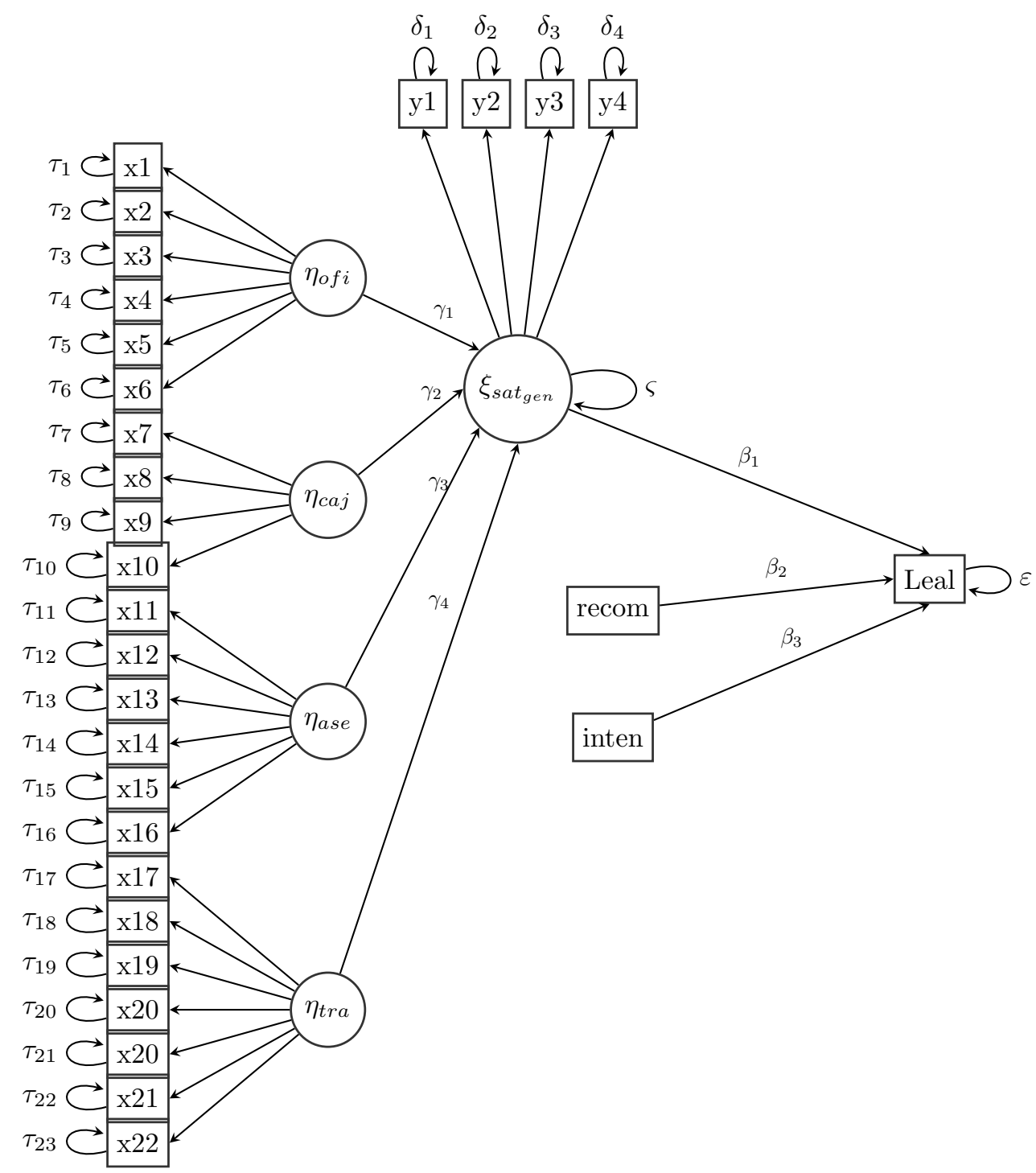

Figura 12: Path Diagram etapa especificación. Fuente: elaboración propia.

Con lo que el modelo estructural está dado por la expresión:

$$
[\xi]=\left[\begin{array}{llll}
\gamma_{1} & \gamma_{2} & \gamma_{3} & \gamma_{4}
\end{array}\right]\left[\begin{array}{l}
\eta_{1} \\
\eta_{2} \\
\eta_{3} \\
\eta_{4}
\end{array}\right]+[\varsigma]
$$

Comunicaciones en Estadística, agosto 2016, Vol. 9, No. 2 
Y finalmente:

$$
\text { Leal }=\left[\begin{array}{lll}
\beta_{1} & \beta_{2} & \beta_{3}
\end{array}\right]\left[\begin{array}{c}
\xi \\
\text { recom } \\
\text { inten }
\end{array}\right]+[\varepsilon]
$$

A primera vista analizando los grados de libertad del modelo, este se encuentra sobreidentificado:

$$
d o f=m(m+1) / 2-2 m-\xi(\xi-1) / 2=400>0
$$

Además, cumple la regla t-rule con la que podemos verificar que no estamos ante un modelo no-identificado:

$$
t \leq\left(\frac{1}{2}\right)(p+q)(p+q+1)
$$

\subsection{Etapa de estimación}

Dada la no normalidad de las variables bajo estudio y su carácter ordinal, se escoge para la estimación una técnica que no haga ningún supuesto sobre la distribución de las variables, de manera que la kurtosis que se presenta no afecte los errores estándar de las estimaciones.

Este método minimiza la función:

$$
F_{D W L S}=[\hat{\rho}-\rho(\theta)]^{\prime} \operatorname{diag}\left(W_{\rho \rho}\right)^{-1}[\hat{\rho}-\rho(\theta)]
$$

Dado que estos métodos buscan reducir la distancia entre las varianzas observadas y el diseño modelado, mediante la matriz $\mathbf{W}$ calculada por los mínimos cuadrados ponderados $(W L S)$, será este precisamente el método de estimación escogido para el modelo, particularmente el método robusto $(D W L S)$ que se basa en las correlaciones policóricas de las variables observadas y que arrojan los resultados que se observan en la figura 13 y en la tabla 8: 


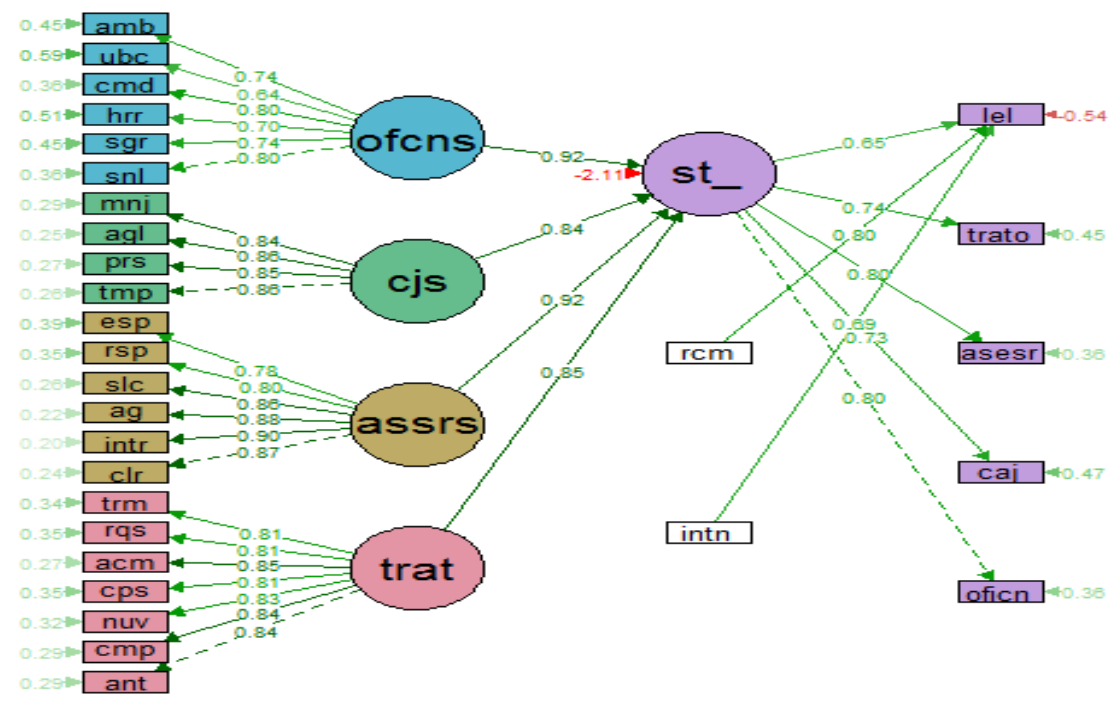

Figura 13: Estimación inicial del modelo. Fuente: elaboración propia.

Los resultados de la figura 13 muestran que la recomendación tiene un mayor efecto sobre la lealtad que el producido por la recompra o la misma satisfacción con el servicio; sin embargo, se observa un problema de especificación del modelo, ya que la varianza del factor satisfacción general es negativa, por lo que se debe especificar de nuevo para evitar este problema. En la tabla 8 se puede observar que en general las variables ingresadas en el modelo son estadísticamente significativas en el cálculo de cada factor.

El efecto total de cada variable se calcula realizando la multiplicación de sus estimaciones, siguiendo en el diagrama la ruta que conecta la variable en cuestión con la lealtad. Los efectos calculados se muestran a en la tabla 9 .

Inicialmente se observa que entre aquellas variables que afectan indirectamente la lealtad, es la relación con los asesores y el factor oficinas las que tienen el mayor efecto indirecto, mientras que a nivel de efecto directo es la recomendación la que tiene mayor trascendencia en la medida de lealtad. 
Tabla 8: Estimaciones iniciales. Fuente: elaboración propia.

\begin{tabular}{|c|c|c|c|c|c|c|}
\hline Variable/factor & Estimate & Std.err & Z-value & $\mathbf{P}(>|z|)$ & Std.lv & Std.all \\
\hline \multicolumn{7}{|l|}{$\begin{array}{l}\text { Regressions: } \\
\text { sat_gene }\end{array}$} \\
\hline oficinas & 1,042 & 0,017 & 60,355 & 0 & 0,924 & 0,924 \\
\hline cajas & 0,59 & 0,009 & 63,886 & 0 & 0,836 & 0,836 \\
\hline asesores & 0,885 & 0,014 & 65,284 & 0 & 0,919 & 0,919 \\
\hline trat & 0,545 & 0,007 & 83,501 & 0 & 0,848 & 0,848 \\
\hline lealtad & & & & & & \\
\hline intenc & 0,585 & 0,02 & 29,922 & 0 & 0,585 & 0,688 \\
\hline recom & 0,761 & 0,028 & 27,443 & 0 & 0,761 & 0,803 \\
\hline sat_gene & 0,958 & 0,009 & 105,588 & 0 & 1,377 & 0,653 \\
\hline \multicolumn{7}{|l|}{ trat $=$} \\
\hline antigue & 1 & & & & 2,237 & 0,842 \\
\hline comporta & 0,956 & 0,011 & 85,695 & 0 & 2,138 & 0,845 \\
\hline nuevo & 0,922 & 0,011 & 85,2 & 0 & 2,062 & 0,828 \\
\hline cupos & 0,845 & 0,01 & 82,581 & 0 & 1,89 & 0,807 \\
\hline acompa & 0,968 & 0,011 & 86,102 & 0 & 2,165 & 0,855 \\
\hline requisit & 0,753 & 0,009 & 82,184 & 0 & 1,683 & 0,807 \\
\hline trami & 0,744 & 0,009 & 81,573 & 0 & 1,665 & 0,812 \\
\hline \multicolumn{7}{|l|}{ asesores $=$} \\
\hline claro & 1 & & & & 1,492 & 0,872 \\
\hline interes & 1,133 & 0,017 & 65,851 & 0 & 1,69 & 0,895 \\
\hline $\mathrm{ag}$ & 1,104 & 0,017 & 65,858 & 0 & 1,647 & 0,883 \\
\hline soluc & 1,148 & 0,017 & 65,895 & 0 & 1,712 & 0,863 \\
\hline respet & 0,747 & 0,012 & 61,478 & 0 & 1,115 & 0,804 \\
\hline espera & 1,148 & 0,017 & 66,513 & 0 & 1,713 & 0,78 \\
\hline \multicolumn{7}{|l|}{ cajas $=$} \\
\hline tiempo & 1 & & & & 2,036 & 0,863 \\
\hline personal & 0,953 & 0,014 & 68,827 & 0 & 1,94 & 0,852 \\
\hline agili & 0,824 & 0,012 & 66,645 & 0 & 1,678 & 0,864 \\
\hline manejo & 0,871 & 0,013 & 67,399 & 0 & 1,773 & 0,843 \\
\hline \multicolumn{7}{|l|}{ oficinas $=$} \\
\hline senaliza & 1 & & & & 1,275 & 0,802 \\
\hline seguridad & 0,899 & 0,015 & 59,536 & 0 & 1,146 & 0,741 \\
\hline horario & 0,92 & 0,015 & 59,539 & 0 & 1,172 & 0,699 \\
\hline comodidad & 1,123 & 0,018 & 61,802 & 0 & 1,431 & 0,802 \\
\hline ubicacion & 0,82 & 0,014 & 58,543 & 0 & 1,045 & 0,64 \\
\hline amabi & 0,823 & 0,014 & 58,096 & 0 & 1,049 & 0,741 \\
\hline \multicolumn{7}{|l|}{ sat_gene $=$} \\
\hline financiera & 0,864 & 0,008 & 109,268 & 0 & 1,243 & $\begin{array}{c}0,001 \\
0,73\end{array}$ \\
\hline atencion & 0,968 & 0,009 & 112,323 & 0 & 1,391 & 0,802 \\
\hline variedad & 1,004 & 0,009 & 112,506 & 0 & 1,444 & 0,745 \\
\hline
\end{tabular}


Tabla 9: Efecto sobre la lealtad. Fuente: elaboración propia.

\begin{tabular}{|l|c|c|c|c|}
\hline Variable & Estimación & ef.indirecto & ef.directo & Total \\
\hline oficinas & 0,924 & 0,603372 & 0 & 0,603372 \\
cajas & 0,836 & 0,545908 & 0 & 0,545908 \\
asesores & 0,919 & 0,600107 & 0 & 0,600107 \\
trat & 0,848 & 0,553744 & 0 & 0,553744 \\
sat gen $_{\text {intenc }}$ & 0,653 & 0 & 0,653 & 0,653 \\
recom & 0,688 & 0 & 0,688 & 0,688 \\
\end{tabular}

\subsection{Diagnóstico de ajuste del modelo}

Una condición de los modelos de ecuaciones estructurales, es que la calidad de su ajuste se mide a través de la capacidad que tienen las estimaciones para reproducir la matriz de covarianzas muestral, o bien, la matriz de correlaciones en el caso de las estimaciones estandarizadas, la cual se deriva a través de $\Sigma(\theta)=\Sigma(\hat{\theta})$, donde $\hat{\theta}=(\Lambda, \eta, \xi)$ es el vector de parámetros que relaciona las covarianzas entre las variables y los parámetros. Si el modelo está bien ajustado, la matriz residual debe ser lo más cercana posible a la matriz nula; para verificar la calidad de este ajuste se deben realizar los pertinentes diagnósticos.

Para esto se han diseñado varias pruebas, las cuales obedecen a motivaciones diferentes, algunas contrastan el ajuste de $\Sigma(\theta)$, algunas otras verifican la parsimonia del modelo y otras comparan el modelo ajustado contra el modelo de base saturado. Sin embargo, existen diferentes situaciones que pueden alterar su resultado, por lo que no siempre se pueden utilizar todas las pruebas; así que en este artículo, antes de escoger alguna con la cual realizar el diagnóstico de ajuste del modelo, se realizan 1000 simulaciones por Bootstrap a diferentes niveles de muestra $(100,200$, $300,400,500,600,700,800,900,1000,1500,2000,2500,3000,3500,4000,4500$, $5000,5500,6000,6500,7000,7500,8000)$ para comprobar su estabilidad (figura 14). 


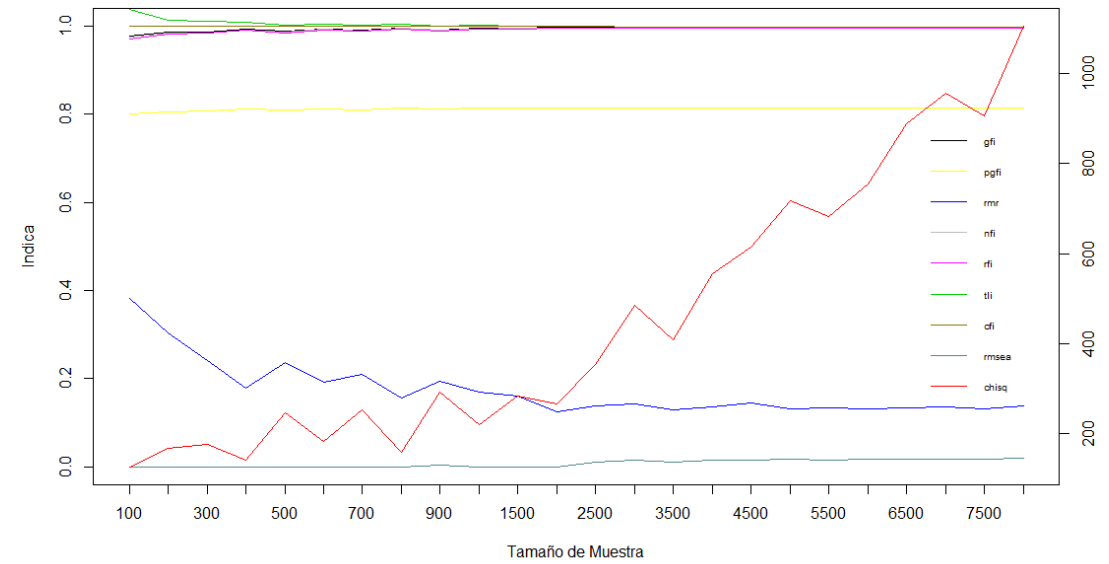

Figura 14: Estabilidad de ajuste a diferentes niveles de muestra. Fuente: elaboración propia.

En la figura 14 se observa que en particular la prueba $\chi^{2}$ aumenta su estadístico a medida que crece el tamaño de la muestra, llevando a rechazar siempre para $n$ grandes; este resultado se encuentra en línea con las investigaciones que han concluido que para muestras grandes pequeñas variaciones entre la matrices de covarianzas muestral y estimada son detectadas como significativas (Bentler \& Bonett 1980).

Con la prueba RMR sucede lo contrario, ya que a medida que aumenta la muestra, sus estimaciones decaen, encontrando su estabilidad para tamaños de muestra superiores a 2.000 casos; por su parte, las pruebas TLI, RFI y GFI alcanzan estabilidad en tamaños de muestra de 500 o 1000 elementos. Se muestran como las más estables las pruebas CFI, RMSEA y PGFI, las cuales contrastan el ajuste de $\Sigma(\theta)$, de residuos del modelo y el contraste contra el modelo saturado ${ }^{2}$.

Tabla 10: Índices de bondad de ajuste. Fuente: elaboración propia.

\begin{tabular}{|l|c|c|c|}
\hline Prueba & Estadística de prueba & Valor óptimo de ajuste & Resultado \\
\hline$\chi^{2}$ & 228551,8671 & $\approx 0,0$ & Mal ajuste \\
cfi & 0,4568 & $\approx 1$ & Mal ajuste \\
rmsea & 0,2624 & $\leq 0,1$ & Mal ajuste \\
pgfi & 0,4522 & $\approx 1$ & Mal ajuste \\
\hline
\end{tabular}

Haciendo un análisis de las pruebas de ajuste del modelo más estables y presentadas en la tabla 10, se encuentra que el modelo inicial no presenta un ajuste adecuado de la matriz de covarianzas muestral.

\footnotetext{
${ }^{2}$ Para una ilustración detallada de las pruebas, ver (Romero 2015)
} 


\subsection{Modificación del modelo}

Los resultados de este modelo muestran un mal ajuste, por lo que procederá a realizar las modificaciones en busca de uno mejor; tales modificaciones buscan liberar parámetros que se fijaron en cero (por ejemplo, la covarianza entre dos variables). Para este fin se utiliza la prueba de multiplicadores de Lagrange (índices de modificación) para maximizar el ajuste del modelo; esta prueba permite establecer las modificaciones que aportaran un mayor cambio en el estadístico de $\chi^{2}$ resultantes de la liberación de estos parámetros restringidos a cero. Sin embargo, establecer estas modificaciones depende básicamente de las implicaciones que sobre la teoría del modelo conlleve dicho cambio, por lo que no todos los cambios son viables, o incluso, algún cambio puede reportar variaciones en la teoría del modelo.

Estas modificaciones se incluyen una a una y tras cada nuevo cambio se revisa de nuevo el ajuste del modelo, hasta encontrar el adecuado. El contraste entre cada modelo se realizará por medio del estadístico LR Test que permite mostrat la diferencia entre las log-verosimilitudes de los modelos (inicial y modificado) mediante la estadística:

$$
L R=-2\left(l\left(\pi^{0} \mid y\right)-l\left(\pi^{1} \mid y\right)\right) \sim \chi_{1}^{2}
$$

Las modificaciones se detienen en el momento que cada liberación de parámetros realizada no genera grandes cambios de $\chi^{2}$ entre los modelos anidados (séptimo modelo en este caso); en la figura 15 se observa que a partir del modelo número siete, los nuevos modelo anidados no genera mayor pérdida de $\chi^{2}$ respecto del modelo anterior, por lo que se introducirán al modelo inicial estos siete cambios, los cuales consisten en la liberación de los parámetros de covarianza entre los factores latentes que afectan de manera indirecta a la satisfacción general.

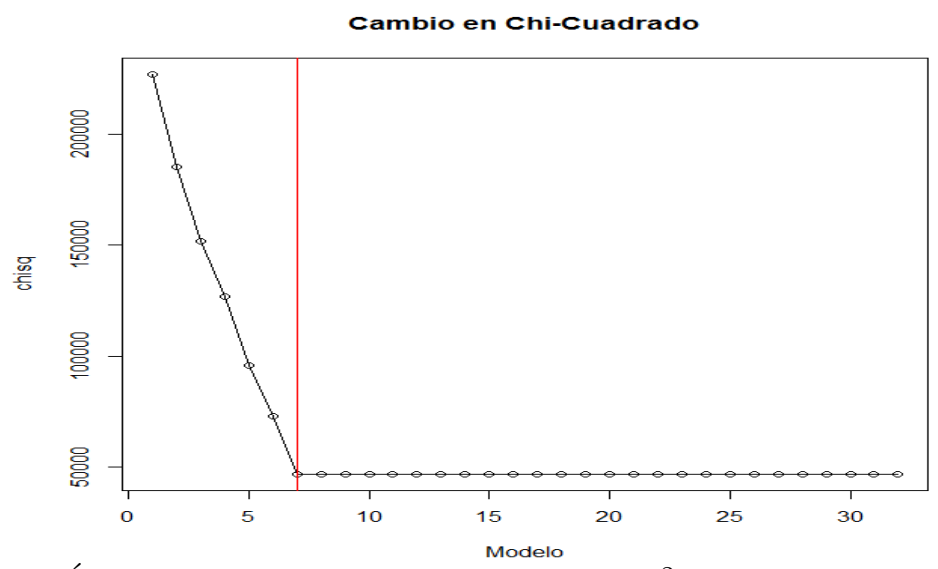

Figura 15: Índices de modificación - Cambios en $\chi^{2}$. Fuente: elaboración propia. 


\subsection{Modelo definitivo}

Verificando los test de bondad de ajuste 11, se observa que finalmente se ha llegado a un buen modelo, tanto en variabilidad total explicada como a nivel de parsimonia y residuos mínimos (tabla 11).

Tabla 11: Índices de bondad de ajuste finales. Fuente: elaboración propia.

\begin{tabular}{|l|c|c|c|}
\hline Prueba & Estadística de prueba & Valor óptimo de ajuste & Resultado \\
\hline chisq & 46935,5 & & \\
cfi & 0,9 & $\approx 1$ & Buen Ajuste \\
rmsea & 0,1 & $\leq 0,1$ & Buen Ajuste \\
pgfi & 0,8 & $\approx 1$ & Buen Ajuste \\
\hline
\end{tabular}

Las estimaciones de los parámetros en la figura 16 permiten observar que las dimensiones de oficinas y asesores son las que más efecto tienen sobre la satisfacción general de los clientes. A la vez, dicha satisfacción afecta en mayor medida la lealtad, lo que se ve reflejado en el coeficiente que tienen esta variable sobre la variable respuesta. Asimismo se observa que la mayor covarianza se encuentra entre los factores oficinas y cajas, por lo que un posible ajuste del modelo que contemple estos dos factores como uno solo podría también tomarse en cuenta.

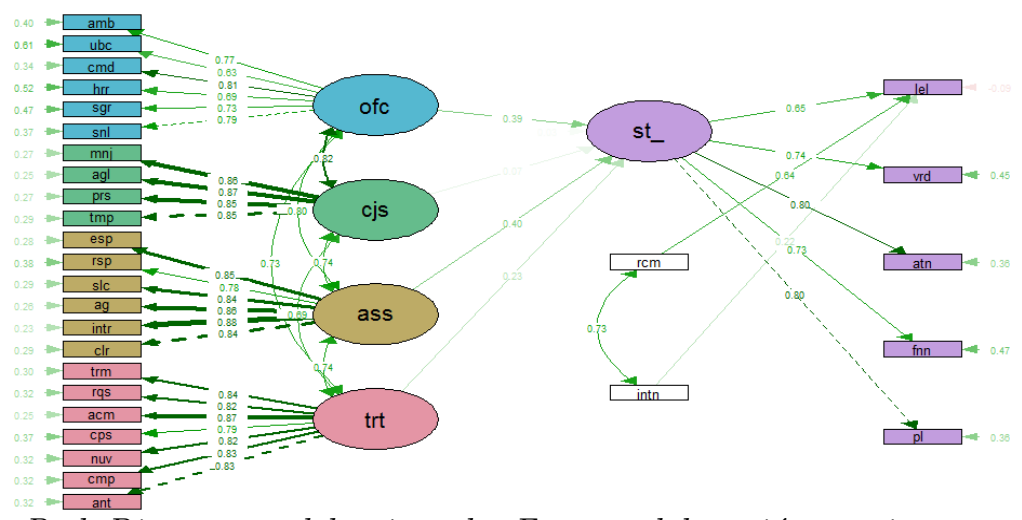

Figura 16: Path Diagram modelo ajustado. Fuente: elaboración propia.

Los resultados en 12 y 13 permiten comprobar el sistema de hipótesis, concluyendo que los factores subyacentes especificados en el modelo tienen un efecto significativo sobre la satisfacción, como también son significativas cada una de las variables que afectan la lealtad de un cliente. También se observa que la relación de covariación entre los factores componentes del servicio es significativa, lo que quiere decir que 
su estudio no se debe hacer de manera aislada como se haría en cualquier modelo de regresión multiple, sino que se deben tener en cuenta las correlaciones existentes entre las diferentes dimensiones del servicio ya que unas afectan a las otras.

Tabla 12: Estimaciones modelo ajustado. Fuente: elaboración propia.

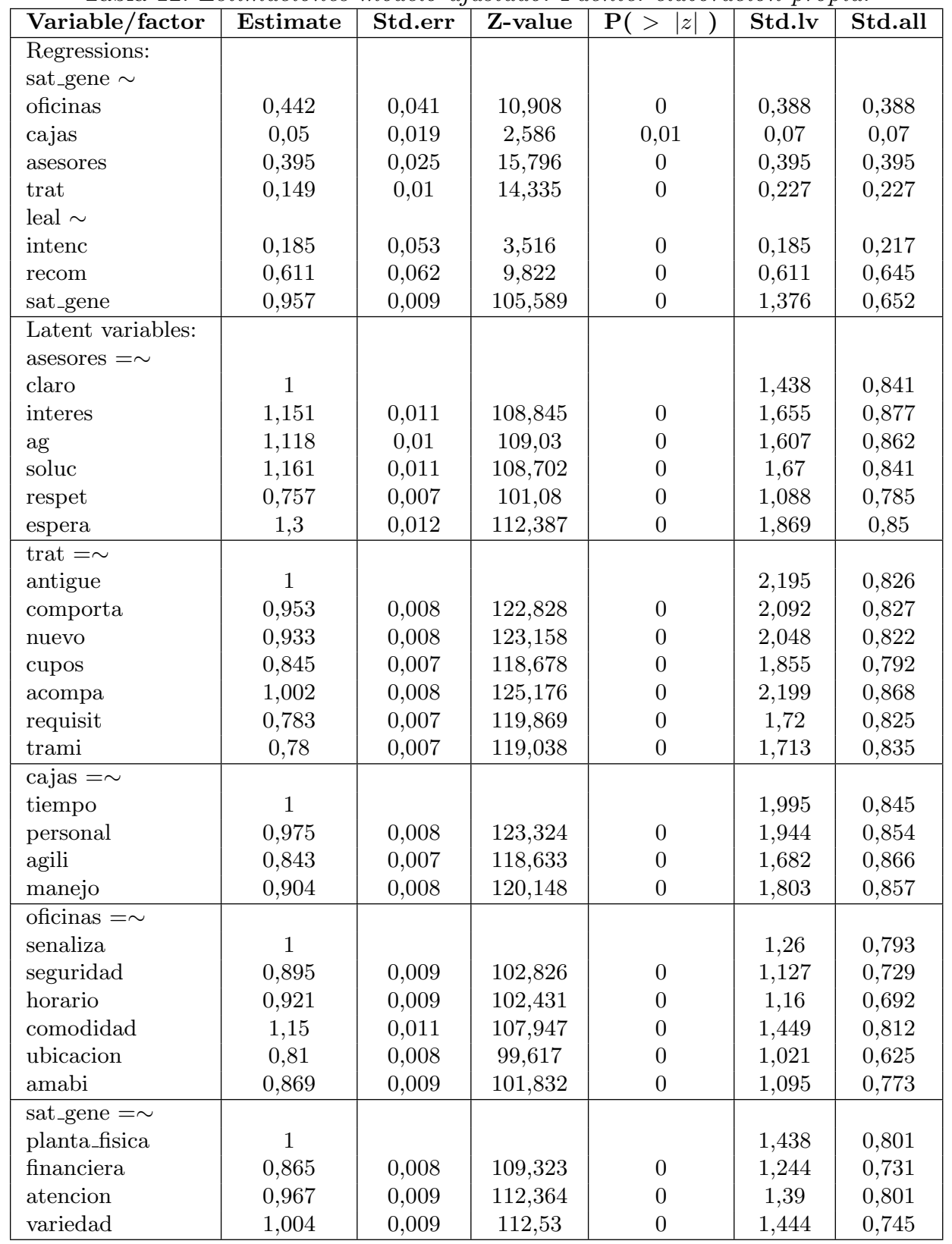


Tabla 13: Estimaciones modelo ajustado (cont.). Fuente: elaboración propia.

\begin{tabular}{|l|c|c|c|c|c|c|}
\hline Variable/factor & Estimate & Std.err & Z-value & $\mathbf{P}(>|z|)$ & Std.lv & Std.all \\
\hline $\begin{array}{l}\text { Covariances: } \\
\text { trat }\end{array}$ & & & & & & \\
asesores & & & & & & \\
oficinas & 2,329 & 0,022 & 106,305 & 0 & 0,738 & 0,738 \\
cajas & 2,012 & 0,019 & 103,253 & 0 & 0,728 & 0,728 \\
$\begin{array}{l}\text { asesores } \\
\text { oficinas } \\
\text { cajas }\end{array}$ & 3,008 & 0,028 & 108,676 & 0 & 0,687 & 0,687 \\
cajas & 1,442 & 0,015 & 94,379 & 0 & 0,796 & 0,796 \\
oficinas \\
intenc \\
recom
\end{tabular}

La expresión matemática del modelo inicial de medida es la siguiente:

$$
\mathbf{X}=\Lambda \eta+\tau
$$

$\left[\begin{array}{c}\text { antigue } \\ \text { comporta } \\ \text { nuevo } \\ \text { cupos } \\ \text { acompa } \\ \text { requisit } \\ \text { trami } \\ \text { claro } \\ \text { interes } \\ \text { ag } \\ \text { soluc } \\ \text { respet } \\ \text { espera } \\ \text { tiempo } \\ \text { personal } \\ \text { agili } \\ \text { manejo } \\ \text { senaliza } \\ \text { seguridad } \\ \text { horario } \\ \text { comodidad } \\ \text { ubicacion } \\ \text { amabi }\end{array}\right]=\left[\begin{array}{cccc}0,826 & 0 & 0 & 0 \\ 0,827 & 0 & 0 & 0 \\ 0,822 & 0 & 0 & 0 \\ 0,792 & 0 & 0 & 0 \\ 0,868 & 0 & 0 & 0 \\ 0,825 & 0 & 0 & 0 \\ 0,835 & 0 & 0 & 0 \\ 0 & 0,841 & 0 & 0 \\ 0 & 0,877 & 0 & 0 \\ 0 & 0,862 & 0 & 0 \\ 0 & 0,841 & 0 & 0 \\ 0 & 0,785 & 0 & 0 \\ 0 & 0,85 & 0 & 0 \\ 0 & 0 & 0,845 & 0 \\ 0 & 0 & 0,854 & 0 \\ 0 & 0 & 0,866 & 0 \\ 0 & 0 & 0,857 & 0 \\ 0 & 0 & 0 & 0,793 \\ 0 & 0 & 0 & 0,729 \\ 0 & 0 & 0 & 0,692 \\ 0 & 0 & 0 & 0,812 \\ 0 & 0 & 0 & 0,625 \\ 0 & 0 & 0 & 0,773\end{array}\right]\left[\begin{array}{c}0,317 \\ \eta_{\text {trato }} \\ \eta_{\text {cajas }} \\ \eta_{\text {oficinas }}\end{array}\right]+\left[\begin{array}{c}0,325 \\ 0,373 \\ 0,247 \\ 0,32 \\ 0,302 \\ 0,294 \\ 0,232 \\ 0,258 \\ 0,292 \\ 0,384 \\ 0,277 \\ 0,285 \\ 0,27 \\ 0,25 \\ 0,265 \\ 0,372 \\ 0,469 \\ 0,521 \\ 0,341 \\ 0,609 \\ 0,402\end{array}\right]$

Comunicaciones en Estadística, agosto 2016, Vol. 9, No. 2 
En tanto que el modelo estructural es:

$$
\begin{aligned}
& {\left[\xi_{\text {satisfacción }}\right]=\left[\begin{array}{llll}
0,369 & 0,292 & -0,098 & 0,231
\end{array}\right]\left[\begin{array}{c}
\eta_{\text {trato }} \\
\eta_{\text {asesores }} \\
\eta_{\text {cajas }} \\
\eta_{\text {oficinas }}
\end{array}\right]+[0,025]} \\
& \text { Leal }=0,65 * \text { satisfacción }+0,64 * \text { recomendación }+0,22 * \text { intención }-0.093
\end{aligned}
$$

\subsection{Impacto de las componentes del servicio sobre la lealtad}

Para la estimación del impacto que tiene cada una de las dimensiones del servicio sobre la lealtad del cliente, se hace uso del efecto total de la variable, el cual se calcula como la suma de los efectos directos e indirectos.

Tabla 14: Efecto final sobre la lealtad del cliente. Fuente: elaboración propia.

\begin{tabular}{|r|c|c|c|c|}
\hline Variable & Estimación & ef.indirecto & ef.directo & Total \\
\hline oficinas & 0,388 & 0,252976 & 0 & 0,252976 \\
cajas & 0,07 & 0,04564 & 0 & 0,04564 \\
asesores & 0,395 & 0,25754 & 0 & 0,25754 \\
trat & 0,227 & 0,148004 & 0 & 0,148004 \\
sat $_{\text {gen }}$ & 0,652 & 0 & 0,652 & 0,652 \\
intenc $_{\text {recom }}$ & 0,217 & 0 & 0,217 & 0,217 \\
rech & 0,645 & 0 & 0,645 & 0,645 \\
\hline
\end{tabular}

En la tabla 14 este se puede observar que dentro de las dimensiones de satisfacción las de mayor efecto son los asesores y las oficinas, en tanto que las cajas tienen el menor impacto en la satisfacción. A nivel global el mayor efecto sobre la lealtad lo tiene la recomendación.

\subsection{Cálculo del indicador de lealtad}

Diferentes enfoques se pueden utilizar para el cálculo del indicador; sin embargo, el propósito de este trabajo es extraer toda la información derivada como base para el cálculo. Se propone realizar el cálculo del indicador de lealtad usando el efecto total de cada una de las componentes del servicio como pesos que ponderan el indicador, de tal manera que:

$$
\text { Lealtad }=\sum_{i=1}^{3} w_{i} v
$$

Donde $w_{i}$ es un vector de ponderaciones construidos con los efectos totales de las variables recom, intenc y el factor sat gen $_{\text {, tal que }}$ 


$$
w_{i}=\frac{\beta_{i}}{\sum_{i=1}^{3} \beta_{i}} \quad \sum_{i=1}^{3} w_{i}=1
$$

y $v$ es una matriz conformada por las variables de satisfacción general $\left(s_{\text {gat }}\right)$, recomendación (recom) e intención de recompra (intenc).

Entonces, la expresión matemática del indicador de lealtad sería:

$$
\begin{gathered}
I_{\text {Lealtad }}=w_{1} * \text { sat }_{\text {gen }}+w_{2} * \text { recom }+w_{3} * \text { intenc } \\
I_{\text {Lealtad }}=0,431 * \text { sat }_{\text {gen }}+0,143 * \text { recom }+0,426 * \text { intenc }
\end{gathered}
$$

\section{Conclusiones}

Las conclusiones son un compendio de lo que ya se mencionó a lo largo de este artículo; sin embargo sintetizando se puede concluir lo siguiente:

- Respecto al mejor método de estimación se encuentra que la función de ajuste por mínimos cuadrados ponderados diagonalizados (DWLS) es la que mejor se ajusta a los datos, ya que es una función libre de distribución y adicionalmente utiliza la matriz de correlaciones policóricas para variables ordinales en la etapa de estimación.

- En su orden, la satisfacción general con el servicio y la recomendación de este son las variables que mayor efecto tienen sobre la lealtad del cliente. Se puede observar igualmente que son los constructos del servicio relacionados a los asesores y las oficinas los que mayor efecto tienen sobre la satisfacción general con el servicio.

- La matriz de acción permite observar que los atributos que componen los factores de asesores y oficinas son los mejor valorados y a la vez son los que mayor efecto tienen sobre la lealtad; sin embargo el atributo espera tiene una calificación inferior al promedio, por lo que con miras a mejorar la lealtad se debe apuntar a subir su calificación promedio. Los atributos componentes del factor cajas presentan en general una satisfacción inferior al promedio y son los que menos efecto tienen sobre la lealtad.

Adicionalmente, durante el desarrollo del presente trabajo surgieron otras inquietudes como por ejemplo el comportamiento de los indicadores de ajuste del modelo y como mejorar el ajuste de este, al respecto, se puede concluir que:

- En los estudios exploratorios generalmente se analiza la manera en que se correlacionan las variables ordinales para formar factores latentes. En estas 
situaciones se debe utilizar la matriz de correlaciones policóricas; sin embargo, la mayoría de paquetes comerciales aún no ha difundido su uso por lo que la realización de un análisis factorial exploratorio podría ser cuestionado. En este trabajo se concluye que para escalas tipo Likert de 10 puntos la matriz de correlaciones policóricas es muy cercana a la matriz de correlación de Pearson, tanto en el valor de la correlación como en su comportamiento, por lo que en estos casos es válido utilizar la matriz de pearson para realizar el análisis factorial en variables ordinales.

- Como estadística de diagnóstico para el ajuste del modelo se encontró que no obstante ser la más popular, la medida $\chi^{2}$ no es robusta ya que se ve afectada de manera directa por el tamaño de la muestra, aumentando su valor a medida que aumenta $n$. En lo relacionado con las medidas de RMR, GFI, NFI, RFI y TLI, encuentran estabilidad en muestras superiores a 1000 casos, por lo que en muchos casos no va a ser permisivo usarlas, dados los costos de levantar muestras grandes. Cabe aclarar que este análisis se realizó sobre el método de estimación DWLS, por lo que en otros métodos de estimación este comportamiento se desconoce.

- Sobre la parte práctica se debe ser riguroso con el análisis descriptivo y validación de supuestos de los datos, ya que aunque no se demuestra directamente en este trabajo, analizando las funciones de ajuste se puede entender fácilmente que los valores de los parámetros cambian dependiendo de la función escogida; sin embargo también se debe tener en cuenta el tamaño de la muestra, ya que los métodos de distribución libre requieren gran cantidad de muestra para poder realizar las estimaciones.

Recibido: 3 de noviembre de 2015 Aceptado: 5 de abril de 2016

\section{Referencias}

Batista, J. \& Coenders, G. (2000), Modelos de Ecuaciones Estructurales, 2 edn, Editorial La Muralla, Madrid, Spain.

Bentler, P. M. \& Bonett, D. G. (1980), 'Significance test and goodness of fit in the analysis of covariance structures', Psychological Bulletin 88(3), 588-606.

Coenders, G., Satorra, A. \& Saris, W. E. (1979), 'Alternative approaches to structural modeling of ordinal data: A Monte Carlo study', Structural Equation Modeling: A Multidisciplinary Journal 4(4), 261-282.

Field, A. (2000), Discovering Statistics using SPSS for Windows, 1 edn, Sage publications, Thousand Oaks, CA.

Gelin, M., Beasley, T. \& Zumbo, B. (2003), 'What is the impact on scale reliability and exploratory factor analysis of a Pearson correlation matrix when some 
respondents are not able to follow the rating scale?'.

*http://faculty.educ.ubc.ca/zumbo/aera/papers/GelinBeasleyZumbo-

7Apr.pdf

Hoyle, R. H. (2012), Handbook of Structural Equation Modeling, 1 edn, The Guilford Press, New York.

Loehlin, J. C. (2004), Latent Variable Models, 4 edn, Laurence Erlbaum Associates Publishers, New Jersey.

Long, J. S. (1983), 'Covariance structure models: an introduction to LISREL', Quantitative Applications in the Social Sciences. SAGE Publications, Inc .

Olsson, U. H. (1979), 'Maximum likelihood estimation of the polychoric correlation coefficient', Psychometrika 44(4), 443-460.

Rietveld, T. \& Van Hout, R. (1993), Statistical Techniques for the Study of Language and Language Behaviour, 1 edn, Mouton de Gruyter, Berlin.

Romero, G. R. (2015), 'Modelo de Lealtad a partir de un análisis de Ecuaciones Estructurales', Tesis de Pregrado. Universidad Santo Tomás .

Saurina, C. (1997), 'Evaluación de un modelo de medida de la calidad en el sector servicios. Las entidades financieras de la comarca de Girona', Annual meeting of the American Educational Research Association (AERA) 39, 219-248.

Streiner, D. (2003), 'Starting at the beginning: an introduction to coefficient alpha and internal consistency', Journal of personality assessment 8(1), 99-103. 